currently one standard-of-care therapy option in women with early, high-risk or locally advanced breast cancer. While some patients respond excellently to preoperative therapy, in other patients significant tumor shrinkage cannot be achieved. We investigated the impact of NAC on circulating immunomodulatory parameters. We also examined whether changes in these parameters correlate with the response to NAC measured by the Residual Cancer Burden (RCB) score determined after neoadjuvant treatment.

Materials and Methods To detect drug-specific effects, two different NAC regimens in primary breast cancer patients scheduled to pre-operative therapy were compared. 39 patients with conventional anthracycline/taxane sequence $(\mathrm{E} / \mathrm{C}->\mathrm{D}$, $n=39)$ and 40 patients with reverse sequence $(D->E / C)$ were included. Blood plasma samples were collected at three time points - 'baseline' (before NAC), 'midterm' (after the first six cycles of NAC) and 'surgery' (after NAC before operation). The plasma levels of uPA, uPAR, TIM-3, MCP-1, MCP-2, OPG, IP-10, CD 27, Eotaxin, Tweak, TRAIL, PD-L2, M-CSF and VEGF-A were determined either by using ELISA or a multiplex bead array immunoassay.

Results OPG, CD27, MCP-1, MCP-2, CCl19, Tweak, TRAIL, PD-L2 and M-CSF decreased between baseline and midterm in $\mathrm{E} / \mathrm{D}->\mathrm{D}$ patients. However, the majority of patients treated with the reverse sequence showed no such effect. These druginduced changes correlated with the RCB score. Non-responders $(\mathrm{RCB} \geq 1.36)$ showed a significantly different pattern than responders.

Conclusion These data confirm that NAC affects the immune system in a drug-specific manner. Factors correlating with the RCB-score might represent promising biomarkers to predict the response to therapy.

Disclosure Information K. Wimmer: None. M. Sachet: None. R. Exner: None. F. Fitzal: None. M. Filipits: None. R. Oehler: None.

\section{P06.09 ANTI-HPSMA CAR ENGINEERED NK-92 CELLS: AN OFF-THE-SHELF CELLULAR THERAPEUTIC FOR TARGETED ELIMINATION OF PROSTATE CANCER CELLS}

${ }^{1} \mathrm{G}$ Zuccolotto*, ${ }^{2} \mathrm{~A}$ Penna, ${ }^{2} \mathrm{IM}$ Montagner, ${ }^{2} \mathrm{D}$ Carpanese, ${ }^{1} \mathrm{~A}$ Rosato. ${ }^{1}$ Università Degli Studi di Padova, Padova, Italy; ${ }^{2}$ Istituto Oncologico Veneto, Padova, Italy

\subsection{6/jitc-2020-ITOC7.88}

Background Adoptive cell therapy of malignant diseases takes advantages of the cellular immune system to recognize and destroy cancer cells. Despite the remarkable success in B cell malignancies after adoptive transfer of CD19 CAR T cells, CAR $\mathrm{T}$ cell therapy in solid tumors has shown less encouraging clinical results, above all caused by tumor escape mechanisms.In order to overcome such limitations, NK-92, a permanent and IL-2-dependent cell line with a high cytotoxicity in vitro, has been engineered in preclinical models with CAR. In this project, we exploited a CAR directed against the human antigen hPSMA that is overexpressed in prostate tumors. This project aimed at transducing NK-92 cell line to obtain a hPSMA-specific CAR NK-92 cell population, to be thereafter characterized in vitro and in vivo for antigen-specific functional activity.

Materials and Methods NK-92 cell line was transduced with a lentiviral vector (LV) carrying a CAR anti-hPSMA. The cell population obtained was then sorted and analyzed for degranulation capacity, IFN $\gamma$ production and lytic activity against hPSMA $^{+}$(PC3-hPSMA, LNCaP) or hPSMA-tumor cell lines. In vivo therapeutic efficacy of CAR-transduced NK-92 was evaluated initially using Winn-Assay and than in subcutaneous and orthotopic tumor models.

Results CAR-expressing LV efficiently transduced NK-92 cells, which in turn produced cytokines, degranulated and exerted a relevant cytotoxic upon challenge with PSMA+ prostate tumor cells, irrespective of $10 \mathrm{~Gy} \gamma$-irradiation. In all the in vivo, tumor models CAR-transduced NK-92 shown a statistically significant inhibition of tumor growth.

Conclusions Chimeric antigen receptor-engineered NK-92 could offer a valid and cost-effective alternative to primary CAR NK or $\mathrm{T}$ cells, in particular in cases, where a suitable donor is not available or the sophisticated infrastructure needed for cell isolation, expansion and genetic modification is missing. This work demonstrates that CAR-engineered NK92 cells display a high and specific recognition of hPSMA+ PC both in vitro as is in vivo, and could represent an efficient strategy as a new therapeutic intervention against prostate carcinoma, thus paving the way to an Off-The-Shelf cellular therapeutic for targeted elimination of cancer cells and induction of protective antitumor immunity.

Disclosure Information G. Zuccolotto: None. A. Penna: None. I.M. Montagner: None. D. Carpanese: None. A. Rosato: None.

\section{P06.10 SHORT TERM INHIBITION OF CHECKPOINT PROTEINS INCREASES EX VIVO EXPANSION OF TUMOUR INFILTRATING LYMPHOCYTES IN HIGH GRADE SEROUS OVARIAN CANCER}

${ }^{1} \mathrm{CA}$ Waddell*, ${ }^{1} \mathrm{MJ}$ Price, ${ }^{1} \mathrm{P}$ Johnson, ${ }^{1,2} \mathrm{RJ}$ Edmondson, ${ }^{1,2} \mathrm{GL}$ Owens. ${ }^{1}$ University of Manchester, Manchester, UK; '2Saint Mary's Hospital, Manchester, UK

\subsection{6/jitc-2020-ITOC7.89}

Background Ovarian cancer is the most lethal gynaecological malignancy, accounting for approximately 185,000 deaths worldwide in 2018. The majority of patients will experience recurrence of disease. Therefore, there is an urgent need for the development of further therapies to improve patient survival. Tumour infiltrating lymphocyte (TIL) therapy has shown clear efficacy in immunogenic cancers, and TIL can be readily expanded ex vivo from samples of high grade serous ovarian cancer (HGSOC). Key indicators of effective TIL products for infusion are high TIL yield and functionality against autologous tumour. Blockade of checkpoint proteins is effective in increasing TIL yield and functional response from ovarian cancer TIL cultures. However, it is unknown whether blockade of other key checkpoints, including programmed death ligand1 (PD-1), T cell immunoglobulin mucin-3 (TIM-3) and lymphocyte activation gene-3 (LAG-3) increase TIL yield in ex vivo cultures from HGSOC samples.

Materials and Methods TIL cultures were generated from surgically resected HGSOC tumour samples and were incubated with CD3/CD28 Dynabeads. 3000IU/mL recombinant interleukin-2 (IL-2) was added on alternate days for 7 days before beads were removed. $1000 \mathrm{IU} / \mathrm{mL}$ IL-2 was added on alternate days for a further 12 days of culture. In cohort $1,10 \mu \mathrm{g} / \mathrm{mL}$ $\alpha$ PD-1, $\alpha$ TIM-3 or $\alpha$ LAG-3 antibodies were added at initiation of TIL cultures only. In cohort $2,10 \mu \mathrm{g} / \mathrm{mL} \alpha \mathrm{PD}-1$, $\alpha$ TIM-3 or $\alpha$ LAG-3 antibodies were added on alternate days 